\title{
New Editor-in-Chief for Quantum Information Processing
}

\author{
Yaakov S. Weinstein
}

Published online: 15 October 2014

(C) Springer Science+Business Media New York 2014

\section{Dear Readers of Quantum Information Processing,}

I would first like to express my appreciation for the honor and privilege to be chosen Editor-in-Chief of Quantum Information Processing (QIP). This position was first held by my own PhD advisor Prof. David Cory and was most recently held by the late Dr. Howard Brandt.

The few months since the untimely passing of Dr. Brandt have been difficult for all of us associated with QIP. Dr. Brandt's seemingly gruff facade easily melted into a heart of gold for all those who knew him. His no-nonsense approach to physics and efficient running of QIP will be missed by all of us. Fortunately, QIP has continued thanks to the dedication of a number of acting editors including Michael Frey, Sam Lomonaco, and John Myers.

I was appointed Editor-in-Chief of QIP a little more than a month ago. Since that time I have had the opportunity to familiarize myself with all aspects of the journal and to speak to many people associated with QIP. During these conversations, the question I found myself facing most often was, "What is the role and purpose of QIP given the large number of journals publishing in the area of quantum information?" I would like to take the opportunity to share my answer with all of you.

Every editor would like to see his or her journal publish the "next big thing," the paper that will change the world and be cited thousands of times. This editor is no exception. However, the role of QIP is much more than to fish for the few big papers. I would like to illustrate this with an insight told to me by Prof. Cory a number of years ago when I was a graduate student. "Yaakov," he said, "there are people out there who think you can count qubits 1,2, one million, but in fact you have to count 1, 2, 3." Of course QIP would like to publish the papers that come up with the great initial ideas,

Y. S. Weinstein $(\varangle)$

MITRE Quantum Information Science Group, Princeton, NJ, USA

e-mail:weinstein@mitre.org 
but we also seek to publish the papers that make the essential contributions from 2 to 3 and then to 4 and so on. My vision as Editor-in-Chief of QIP is that in the future people will look back at the early history of quantum information and say, "There were good ideas in lots of journals, but we could not have turned them into reality without QIP."

Part of this vision requires teamwork between authors, reviewers, and editors. Not every manuscript submitted to QIP deserves to be published, but every manuscript deserves fair reviews made by reviewers (or editors) seeking to help the authors, not shoot them down. Even in the short time I have acted as editor I have been amazed by some of the excellent contributions made by our reviewers. Though QIP recognizes our top reviewers, these reviewers will never receive the complete credit they deserve for turning a rough stone into a diamond. I would like this attitude to be synonymous with QIP.

QIP will continue to publish quality work in areas which are currently our strengths, such as quantum cryptography and communications. However, my vision of QIP requires that we also do our best to improve areas in which we are not as strong, such as experimental realizations of quantum information processing. To accomplish this, I will be working with several not yet appointed associate editors. These associate editors and I will be carefully studying all aspects of our journal to identify areas for improvement.

In all of these tasks, I will also look to the Editorial Board for ideas and inspiration. We have an extremely strong and dedicated Editorial Board and I have had the good fortune of working with some of them and learning from each of them via their talks and articles.

Finally, I would like to encourage QIPs most valuable resource, its readers and contributors, to forward your own suggestions and constructive criticisms. As with all scientific journals, QIP exists to broadcast and publicize research results and investigations. Please help us accomplish this by letting me know in what ways you think the journal can be improved.

My best wishes for continued success in all of your endeavors and I look forward to hearing from each of you,

Yaakov S. Weinstein

Editor-in-Chief

Quantum Information Processing 